\title{
VARIATION OF THE DISCRETE EIGENVALUES OF NORMAL OPERATORS
}

\author{
L. ELSNER AND S. FRIEDLAND
}

(Communicated by Palle E. T. Jorgensen)

\begin{abstract}
The Hoffman-Wielandt inequality, which gives a bound for the distance between the spectra of two normal matrices, is generalized to normal operators $A, B$ on a separable Hilbert space, such that $A-B$ is HilbertSchmidt.
\end{abstract}

\section{INTRODUCTION}

Let $A, B$ be two $n$-by- $n$ normal matrices. The famous Hoffman-Wielandt inequality states that the eigenvalues $\lambda_{i}, i=1, \ldots, n$, of $A$ and $\mu_{i}, i=$ $1, \ldots, n$, of $B$ can be ordered in such a way that

$$
\sum_{i=1}^{n}\left|\lambda_{i}-\mu_{i}\right|^{2} \leq\|A-B\|_{F}^{2},
$$

where

$$
\|C\|_{F}^{2}=\sum_{i, j=1}^{n}\left|c_{i j}\right|^{2}
$$

is the Euclidean or Hilbert-Schmidt norm of a matrix $C=\left(c_{i j}\right)$.

Several generalizations of this result have appeared in the literature.

Recently, Bhatia and Bhattacharya [B-B] obtained an analogous result for the joint eigenvalues of $k$-tuples of commuting normal matrices; see also Elsner [Els] for a simplified proof. In this note we will concentrate on the infinite dimensional case.

Let $H$ be an infinite dimensional separable Hilbert space and $A: H \rightarrow H$ a bounded linear operator. We call a sequence $\alpha=\left(\alpha_{1}, \alpha_{2}, \ldots\right)$ of complex numbers an extended enumeration of the discrete spectrum of $A$ if all discrete eigenvalues of $A$ (i.e., isolated points of the spectrum with finite multiplicity as eigenvalues) belong to $\alpha$ (counted with their multiplicity), and all other elements of $\alpha$ are in the essential spectrum, i.e., they are limits of discrete eigenvalues, eigenvalues of infinite multiplicity, or in the continuous spectrum. Let $H$ be an infinite dimensional Hilbert space (not necessary separable) and

Received by the editors January 10, 1994.

1991 Mathematics Subject Classification. Primary 47A55, 47B10, 47B15.

(c)1995 American Mathematical Society 
$A: H \rightarrow H$ be a bounded normal operator. $A$ is called diagonal if $H$ has an orthonormal basis which consists entirely of eigenvectors of $A$.

Kato [Kat] has shown that for $A, B$ hermitian there exist extended enumerations $\left\{\alpha_{i}\right\},\left\{\beta_{i}\right\}$ of the discrete spectra of $A, B$ such that

$$
\sum_{i=1}^{\infty}\left|\alpha_{i}-\beta_{i}\right|^{2} \leq\|A-B\|_{F}^{2}
$$

provided that $A-B$ is a Hilbert-Schmidt operator. Here a compact operator $C: H \rightarrow H$ is Hilbert-Schmidt if the singular values $s_{j}(C), j=1,2, \ldots$, satisfy

$$
\|C\|_{F}^{2} \equiv \sum_{j=1}^{\infty} s_{j}^{2}(C)<\infty .
$$

Kato's result (1.2) was extended by Bhatia-Sinha [B-S] to unitary $A, B$. Bhatia-Elsner in [B-E] showed that (1.2) holds for $A, B$ normal HilbertSchmidt operators, see also $[\mathrm{C}-\mathrm{H}]$. Here the essential spectrum is the point $z=0$.

In this note we relax their assumptions by requiring $A, B$ only to be normal with $A-B$ Hilbert-Schmidt.

Our main tool is Theorem 1. In the finite dimensional case it is a straightforward consequence of Birkhoff's theorem that the extreme points of the doubly stochastic matrices are the permutation matrices. In our case we have to use a selection procedure different from the one used in [B-E].

An immediate consequence is our first result (Theorem 2):

If $A$ and $B$ both are bounded diagonal operators, then there exist extended enumerations $\left\{\alpha_{i}\right\},\left\{\beta_{i}\right\}$ of the discrete spectra of $A$ and $B$ such that (1.2) holds.

Weyl in [Wey] proved that each bounded hermitian operator in a separable Hilbert space is a hermitian diagonal operator plus a compact one. J. von Neumann proved in [Neu] that the compact operator can be chosen to be Hilbert-Schmidt with an arbitrary small Hilbert-Schmidt norm. Later on Berg showed in [Ber] that any normal operator $A$ is a diagonal operator plus a compact one. If in addition the spectrum of $A$ lies on a rectifiable curve, then the compact operator can be chosen to be Hilbert-Schmidt with an arbitrary small Hilbert-Schmidt norm. Finally, Voiculescu in [Voi] proved that any bounded normal operator in a separable Hilbert space is a bounded diagonal operator plus a Hilbert-Schmidt operator with an arbitrary small HilbertSchmidt norm.

In Theorem 3 we derive (1.2) for any bounded normal operators $A, B$ so that $A-B$ is Hilbert-Schmidt, using Voiculescu's result.

\section{Diagonal operators}

Let $\left\{\alpha_{i}\right\}_{i \in \mathbb{N}}$ be a sequence of complex numbers. A sequence $\left\{\alpha_{i}^{\prime}\right\}_{i \in \mathbb{N}}$ is called an extended enumeration of $\left\{\alpha_{i}\right\}$, if each $\alpha_{i}^{\prime}$ is either an element of $\left\{\alpha_{i}\right\}_{i \in \mathbb{N}}$ or an accumulation point of this sequence and each $\alpha_{i}, i \in \mathbb{N}$, appears among the $\left\{\alpha_{i}^{\prime}\right\}_{i \in \mathbb{N}}$ with at least the same multiplicity as in the original sequence. Higher multiplicity can only occur if $\alpha_{i}$ is an accumulation point of the original sequence. 
Theorem 1. Let $\left(\alpha_{i j}\right), i, j \in \mathbb{N}$, denote an infinite doubly stochastic matrix, i.e.,

$$
\alpha_{i j} \geq 0, \quad \sum_{k} \alpha_{i k}=1, \quad \sum_{k} \alpha_{k j}=1 \quad \text { for all } i, j \in \mathbb{N} .
$$

Let $\left\{\lambda_{i}\right\}_{i \in \mathbb{N}},\left\{\mu_{j}\right\}_{j \in \mathbb{N}}$ be two bounded sequences of complex numbers such that

$$
\sum_{i, j} \alpha_{i j}\left|\lambda_{i}-\mu_{j}\right|^{2}=K^{2}<\infty .
$$

Then there are extended enumerations $\left\{\lambda_{i}^{\prime}\right\}$ of $\left\{\lambda_{i}\right\}$ and $\left\{\mu_{i}^{\prime}\right\}$ of $\left\{\mu_{i}\right\}$ such that

$$
\sum_{i=1}^{\infty}\left|\lambda_{i}^{\prime}-\mu_{i}^{\prime}\right|^{2} \leq K^{2}
$$

Proof. Let $A_{n}$ denote the $n$-by- $n$ doubly substochastic matrix formed by the first $n$ rows and columns of $\left(\alpha_{i j}\right)$ :

$$
A_{n}=\left(\alpha_{i j}\right)_{1}^{n} \text {. }
$$

If we define $r_{i}^{(n)}=\sum_{j=1}^{n} \alpha_{i j} \leq 1, c_{i}^{(n)}=\sum_{j=1}^{n} \alpha_{j i} \leq 1$, then the $2 n$-by-2n matrix

$$
\left(\begin{array}{cc}
A_{n} & \operatorname{diag}\left(1-r_{i}^{(n)}\right) \\
\operatorname{diag}\left(1-c_{i}^{(n)}\right) & A_{n}^{T}
\end{array}\right)=\left(b_{i j}^{(n)}\right)
$$

is doubly stochastic and hence convex combination of permutation matrices $P_{\pi}=\left(\delta_{\pi(i), j}\right)_{i, j=1}^{2 n}$. Obviously, if $P_{\pi}$ appears explicitly in this convex combination, then $b_{i \pi(i)}>0$ for all $i=1, \ldots, 2 n$.

Let

$$
x_{i j}^{(n)}= \begin{cases}\left|\lambda_{i}-\mu_{j}\right|^{2}, & i, j \leq n, \\ \inf _{k>n}\left|\lambda_{i}-\mu_{k}\right|^{2}, & i \leq n, j=n+i, \\ \inf _{k>n}\left|\lambda_{k}-\mu_{j}\right|^{2}, & j \leq n, i=n+j, \\ 0 & \text { else. }\end{cases}
$$

Then (2.1) implies

$$
\sum_{i, j=1}^{2 n} b_{i j}^{(n)} x_{i j}^{(n)} \leq K^{2}
$$

and hence there exists a permutation $\sigma_{n}$ of $\{1, \ldots, 2 n\}$ such that

$$
\sum_{i=1}^{2 n} x_{i, \sigma_{n}(i)}^{(n)} \leq K^{2}
$$

In addition $b_{i, \sigma_{n}(i)}^{(n)}>0, \quad i=1, \ldots, 2 n$.

We observe that for $i \leq n$ either $\sigma_{n}(i) \leq n$ or $\sigma_{n}(i)=n+i$. Similarly $\sigma_{n}(i) \leq n$ implies $i \leq n$ or $i=n+\sigma_{n}(i)$. Introducing the sets

$$
\begin{gathered}
U_{n}=\{1, \ldots, n\} \cap \sigma_{n}^{-1}(\{1, \ldots, n\}), \quad U_{n}^{\prime}=\{1, \ldots, n\} \backslash U_{n} \\
V_{n}=\sigma_{n}\left(U_{n}\right), \quad V_{n}^{\prime}=\{1, \ldots, n\} \backslash V_{n},
\end{gathered}
$$


we can write $(2.6)$ as

$$
\sum_{i \in U_{n}}\left|\lambda_{i}-\mu_{\sigma_{n}(i)}\right|^{2}+\sum_{i \in U_{n}^{\prime}}\left|\lambda_{i}-\mu_{\varphi_{n}(i)}\right|^{2}+\sum_{j \in V_{n}^{\prime}}\left|\mu_{j}-\lambda_{\psi_{n}(j)}\right|^{2} \leq K^{2}
$$

where the $\mu_{\varphi_{n}(i)} \in \bar{M}$, the closure of $M=\left\{\mu_{j}: j \in \mathbb{N}\right\}$, are such that

$$
\left|\lambda_{i}-\mu_{\varphi_{n}(i)}\right|=\inf _{k>i}\left|\lambda_{i}-\mu_{k}\right|
$$

and similarly the $\lambda_{\psi_{n}(j)} \in \bar{\Lambda}, \quad \Lambda=\left\{\lambda_{i}: i \in \mathbb{N}\right\}$, are such that

$$
\left|\mu_{j}-\lambda_{\psi_{n}(j)}\right|=\inf _{k>j}\left|\mu_{j}-\lambda_{k}\right| \text {. }
$$

So for $n=1,2 \ldots$ we have constructed mappings

$$
\rho_{n}:\{1, \ldots, n\} \rightarrow \bar{M}, \quad \tau_{n}:\{1, \ldots, n\} \rightarrow \bar{\Lambda}
$$

by

which satisfy

$$
\rho_{n}(i)=\left\{\begin{array}{ll}
\mu_{\sigma_{n}(i)}, & i \in U_{n}, \\
\mu_{\varphi_{n}(i)}, & i \in U_{n}^{\prime},
\end{array} \quad \tau_{n}(i)= \begin{cases}\lambda_{\sigma_{n}^{-1}}(i), & i \in V_{n}, \\
\lambda_{\psi_{n}(i)}, & i \in V_{n}^{\prime},\end{cases}\right.
$$

$$
\sum_{i \in U_{n}}\left|\lambda_{i}-\rho_{n}(i)\right|^{2}+\sum_{i \in U_{n}^{\prime}}\left|\lambda_{i}-\rho_{n}(i)\right|^{2}+\sum_{j \in V_{n}^{\prime}}\left|\mu_{j}-\tau_{n}(j)\right|^{2} \leq K^{2} .
$$

We now construct extended matchings $\left\{\lambda_{i}^{\prime}\right\},\left\{\mu_{i}^{\prime}\right\}$ by the following process:

We start with $i=1$ and set $\lambda_{i}=\lambda_{1}^{\prime}$. If the sequence $\left\{\rho_{n}(1), n=1,2, \ldots\right\}$ contains a constant subsequence $\rho_{\nu_{n}}(1)=\mu, n \in \mathbb{N}$, where $\mu=\mu_{j}$ and $j$ is minimal, then we set $\mu_{1}^{\prime}=\mu_{j}$. Otherwise we choose a convergent subsequence $\rho_{\nu_{n}}(1) \rightarrow \mu_{\infty} \in \bar{M}$ and set $\mu_{1}^{\prime}=\mu_{\infty}$. For convenience of notation we replace $\nu_{n}$ by $n$ in the following.

If $\mu_{1} \neq \mu_{1}^{\prime}$ we set $\mu_{1}=\mu_{2}^{\prime}$. We consider now the sequence $\left\{\tau_{n}(1)\right\}$. If it contains a constant subsequence $\tau_{\nu_{n}}(1)=\lambda_{j}$, then necessarily $j \neq 1$. If $j$ is the minimal such index, we set $\lambda_{2}^{\prime}=\lambda_{j}$. Otherwise we choose a convergent subsequence $\tau_{\nu_{n}}(1) \rightarrow \lambda_{\infty} \in \bar{\Lambda}$ and set $\lambda_{2}^{\prime}=\lambda_{\infty}$.

Proceeding in this manner, extended enumerations $\lambda_{i}^{\prime}, \mu_{i}^{\prime}$ are constructed. For any finite sum we have

$$
\sum_{j=1}^{m}\left|\lambda_{i}^{\prime}-\mu_{i}^{\prime}\right|^{2} \leq K^{2}
$$

which follows from (2.8), if we let there $n \rightarrow \infty$.

This proves the theorem.

As an application of Theorem 1 we prove now the following generalization of the Hoffman-Wielandt theorem.

Theorem 2. Let $A, B: H \rightarrow H$ be two bounded diagonal operators mapping a separable Hilbert space $H$ into itself. Let $\left\{\lambda_{i}\right\},\left\{\mu_{j}\right\}$ be the eigenvalues of $A$ and $B$ resp. counted with their multiplicites. Assume that $B-A$ is a HilbertSchmidt operator. Then there are extended enumerations of the spectra $\left\{\lambda_{i}\right\}$ and $\left\{\mu_{i}\right\}$, called $\left\{\lambda_{i}^{\prime}\right\}$ and $\left\{\mu_{i}^{\prime}\right\}$, such that

$$
\sum_{i=1}^{\infty}\left|\lambda_{i}^{\prime}-\mu_{i}^{\prime}\right|^{2} \leq\|B-A\|_{F}^{2}
$$


Proof. We recall (see e.g. [G-K], p. 106) that a bounded compact linear operator from $H$ into itself is called a Hilbert-Schmidt operator, if $\sum_{j=1}^{\infty} s_{j}^{2}(C)=$ $\|C\|_{F}^{2}<\infty$, where the $s_{j}$ are the singular values of $C$.

Then for any complete orthonormal system $\left\{\varphi_{i}\right\}$ one has (e.g. [G-K], p. 107)

$$
\|C\|_{F}^{2}=\sum_{i=1}^{\infty}\left\|C \varphi_{i}\right\|^{2}
$$

As $A, B$ are diagonal, there are complete orthonormal systems of eigenvectors $\left\{e_{i}\right\},\left\{f_{j}\right\}$ such that

$$
A e_{i}=\lambda_{i} e_{i} \quad B f_{j}=\mu_{j} f_{j} \quad i, j=1,2, \ldots
$$

As $A e_{i}=\sum_{j=1}^{\infty}\left(A e_{i}, f_{j}\right) f_{j}$, we have

$$
\left\|A e_{i}\right\|^{2}=\sum_{j=1}^{\infty}\left|\left(A e_{i}, f_{j}\right)\right|^{2} .
$$

It follows that

$$
\begin{aligned}
\|A-B\|_{F}^{2} & =\sum_{i}\left\|(A-B) e_{i}\right\|^{2}=\sum_{i, j}\left|\left((A-B) e_{i}, f_{j}\right)\right|^{2} \\
& =\sum_{i, j=1}^{\infty}\left|\left(\lambda_{i}-\mu_{j}\right)\left(e_{i}, f_{j}\right)\right|^{2}=\sum_{i, j}\left|\lambda_{i}-\mu_{j}\right|^{2} \alpha_{i j}, \quad \alpha_{i j}=\left|\left(e_{i}, f_{j}\right)\right|^{2}
\end{aligned}
$$

where $\left(\alpha_{i j}\right)$ is an infinite doubly stochastic matrix. The claim follows now from Theorem 1.

\section{THE GeNERAL CASE}

Theorem 3. Let $A, B: H \rightarrow H$ be two bounded normal operators, where $H$ is a separable Hilbert space. Assume that $A-B$ is Hilbert-Schmidt. Then there exist extended enumerations $\left\{\lambda_{i}^{\prime}\right\},\left\{\mu_{i}^{\prime}\right\}$ of the spectra of $A$ and $B$, resp., such that

$$
\sum_{i=1}^{\infty}\left|\lambda_{i}^{\prime}-\mu_{i}^{\prime}\right|^{2} \leq\|A-B\|_{F}^{2} .
$$

Proof. By [Voi] there are sequences of normal bounded operators $A_{i}, B_{i}, \quad i=$ $1,2, \ldots$, such that

$$
\lim \left\|A_{i}-A\right\|_{F}=\lim \left\|B_{i}-B\right\|_{F}=0
$$

and $A_{i}, B_{i}$ are diagonal.

Hence by Theorem 2 there are enumerations of the spectra of $A_{i}, B_{i}$, namely $\left\{\lambda_{\nu, i}^{\prime}\right\}_{\nu=1}^{\infty},\left\{\mu_{\nu, i}^{\prime}\right\}_{\nu=1}^{\infty}$, such that

$$
\sum_{\nu=1}^{\infty}\left|\lambda_{\nu, i}^{\prime}-\mu_{\nu, i}^{\prime}\right|^{2} \leq\left\|A_{i}-B_{i}\right\|_{F}^{2}, \quad i=1,2, \ldots
$$

We will now construct an extended enumeration of the spectrum of $A, B$ satisfying (3.1). 
Let $\left\{\lambda_{1}, \lambda_{2}, \ldots\right\}=\Lambda$ denote the (possibly finite) point spectrum of $A$, similarly $\left\{\mu_{1}, \mu_{2}, \ldots\right\}=M$ that of $B$.

We set $\lambda_{1}^{\prime}=\lambda_{1}$, if $\Lambda \neq \varnothing$. As $A_{i} \rightarrow A$ there is a sequence of eigenvalues $\lambda^{(i)}$ of $A_{i}$ converging to $\lambda_{1}$. As those appear as some $\lambda_{1_{\nu}, i}^{\prime}$ we can permute the enumerations of $A_{i}, B_{i}$ so that $\lambda_{1, i}^{\prime} \rightarrow \lambda_{1}$. We consider the set of accumulation points of $\left\{\mu_{1, i}^{\prime}\right\}$. If this contains an element in $M$, say $\mu_{j}$ with $j$ minimal, we set $\mu_{1}^{\prime}=\mu_{j}$. If not, then we choose any limit point $\xi \in \sigma(B)$ and set $\mu_{1}^{\prime}=\xi$. From

$$
\left|\lambda_{i, 1}^{\prime}-\mu_{i, 1}^{\prime}\right| \leq\left\|A_{i}-B_{i}\right\|_{F}
$$

we have

$$
\left|\lambda_{1}^{\prime}-\mu_{1}^{\prime}\right| \leq\|A-B\|_{F}
$$

By eventually going over to a subsequence, we have that

$$
\lim _{i \rightarrow \infty} \lambda_{1, i}^{\prime}=\lambda_{1}^{\prime}, \quad \lim _{i \rightarrow \infty} \mu_{1, i}^{\prime}=\mu_{1}^{\prime} .
$$

If now $\mu_{1}^{\prime} \neq \mu_{1}$, and there are still elements in $\Lambda \cup M$ which do not yet appear as elements in $\left\{\lambda_{1}^{\prime}, \lambda_{2}^{\prime}, \ldots\right\}$ or $\left\{\mu_{1}^{\prime}, \mu_{2}^{\prime}, \ldots\right\}$, we proceed as with $\lambda_{1}$. In this way we construct (as long as $\Lambda \cup M$ is infinite) sequences $\left\{\lambda_{1}^{\prime}, \lambda_{2}^{\prime}, \ldots\right\}$, $\left\{\mu_{1}^{\prime}, \mu_{2}^{\prime}, \ldots\right\}$ such that of each pair $\left(\lambda_{i}^{\prime}, \mu_{i}^{\prime}\right)$ at least one element is some $\lambda_{\nu}$ or some $\mu_{\nu}$ and the whole sequences $\left\{\lambda_{i}\right\},\left\{\mu_{i}\right\}$ are exhausted. Also in each step

$$
\sum_{\nu=1}^{r}\left|\lambda_{\nu}^{\prime}-\mu_{\nu}^{\prime}\right|^{2} \leq\|A-B\|_{F}^{2}
$$

as this can be written as a suitable limit of expressions, which by (3.3) are bounded by $\left\|A_{i}-B_{i}\right\|_{F}^{2}$.

We remark also that when $N=|\Lambda \cup M|$ is finite, this construction yields a finite sum of length $\ell \leq N$, which can be formally extended to an infinite sum as on the left-hand side of (3.1) by setting $\lambda_{i}^{\prime}=\mu_{i}^{\prime}=k$ for all $i \geq \ell+1$, where $k$ is some element in the essential spectrum of $A$. Here we use the fact that the essential spectrum of $A$ coincides with that of $B$ (see e.g. [Kat] or [Wey]).

\section{FinAl Remarks}

The first two remarks discuss our assumptions.

The underlying Hilbert space $H$ has been assumed to be separable. This is no serious restriction under the condition that $A-B$ is Hilbert-Schmidt. If $H$ is a general Hilbert space and $A-B$ is Hilbert-Schmidt, then we can see that $A=B$ except on a separable closed subspace $H^{\prime} \subset H$, which in addition is invariant under $A$ and $B, A\left(H^{\prime}\right) \subset H^{\prime}, B\left(H^{\prime}\right) \subset H^{\prime}$.

The second remark concerns eigenvalues of $A$ (resp. $B$ ) of infinite multiplicity. They are not necessarily included in an extended enumeration of the discrete spectrum. However, as they are in the essential spectrum, which is invariant under Hilbert-Schmidt perturbations, they are also in the essential spectrum of $B$ (resp. $A$ ). Hence we could easily include those points in the extended enumeration without violating (1.2). 
It is also proved in $[\mathrm{H}-\mathrm{W}]$ that one can order the eigenvalues of $A, B$ so that

$$
\sum_{i=1}^{n}\left|\lambda_{i}-\mu_{i}\right|^{2} \geq\|A-B\|_{F}^{2} .
$$

As pointed out in [B-E], this carries over to the case that $A, B$ both are normal and Hilbert-Schmidt. Also under the assumptions on this note such a dual result can be established. It is easy to see that Theorem 1 carries over to show the existence of enumerations satisfying (2.2) with reverse inequality sign.

Similarly it should be possible to generalize the results on joint eigenvalues of tuples of commuting matrices to the case of bounded diagonal commuting operators (see $[\mathrm{B}-\mathrm{B}]$ and $[\mathrm{Els}])$.

\section{ACKNOWLEDGMENT}

We would like to thank Paul Halmos for the reference [Ber] and Rajendra Bhatia for the reference [Voi].

\section{REFERENCES}

[Ber] I. D. Berg, An extension of the Weyl-von Neumann theorem to normal operators, Trans. Amer. Math. Soc. 160 (1971), 365-371.

[B-B] R. Bhatia and T. Bhattacharya, A generalization of the Hoffman-Wielandt theorem, Linear Algebra Appl. 179 (1993), 11-17.

[B-E] R. Bhatia and L. Elsner, The Hoffman-Wielandt inequality in infinite dimensions, Proc. Indian Acad. Sci. Math. Sci. 104 (1994), 483-494.

[B-S] R. Bhatia and K.B. Sinha, A unitary analogue of Kato's theorem on variation of discrete spectra, Lett. Math. Phys. 15 (1988), 201-204.

[C-H] J. A. Cochran and E. W. Hinds, Improved error bounds for the eigenvalues of certain normal operators, SIAM J. Numer. Anal. 9 (1972), 446-453.

[Els] L. Elsner, A note on the Hoffman-Wielandt theorem, Linear Algebra Appl. 182 (1993), 235-237.

[Fri] S. Friedland, Inverse eigenvalue problems, Linear Algebra Appl. 17 (1977), 15-51.

[G-K] I. C. Gohberg and M. G. Krein, Introduction to the theory of linear nonselfadjoint operators, Amer. Math. Soc., Providence, RI, 1968.

[H-W] A. J. Hoffman and H. W. Wielandt, The variation of the spectrum of a normal matrix, Duke Math. J. 20 (1953), 37-39.

[Kat] T. Kato, Variation of discrete spectra, Comm. Math. Phys. 111 (1987), 501-504.

[Neu] J. von Neumann, Charakterisierung des Spektrums eines Integraloperators, Actualités Sci. Indust., vol. 229, Hermann, Paris, 1935; reprinted in his Collected Works, Vol. IV, Pergamon Press, Oxford, 1962, pp. 38-59.

[Voi] D. Voiculescu, Some results on norm-ideal perturbations of Hilbert space operators, J. Operator Theory 2 (1979), 3-37.

[Wey] H. Weyl, Über beschränkte quadratische Formen, deren Differenz vollstetig ist, Rend. Circ. Mat. Palermo 27 (1909), 373-392.

FakUltät für Mathematik, Universität Bielefeld, Postfach 100131, 33501 Bielefeld, GeRMANY

E-mail address: elsner@mathematik.uni-bielefeld.de

Department of Mathematics, Statistics, and Computer Science, University of Illinois at Chicago, Chicago, Illinois 60680

E-mail address: u12735Quicvm.bitnet 